\title{
Riesgos laborales en trabajadores latinoamericanos del sector agrícola: Una revisión sistemática
}

\author{
Job related risks in agriculture workers from Latin America: a systematic review
}

Johana Madelyn Matabanchoy-Salazar1* orcid.org/0000-0002-3831-7252

Fátima Díaz-Bambula² orcid.org/0000-0002-7663-403X

1. Departamento de Psicología, Universidad de Nariño. Pasto, Colombia.

2. Facultad de Psicología, Universidad del Valle. Cali, Colombia.

\section{Resumen}

Introducción: Lineamientos establecidos por la Organización Internacional del Trabajo (OIT), buscan promover alianzas intersectoriales en relación a la salud y seguridad en el trabajo del sector agrícola. Objetivo: Identificar los riesgos laborales y sus consecuencias en la salud de trabajadores de países latinoamericanos a partir de una revisión sistemática en el periodo 2010 - 2020. Materiales y métodos: Con base en la guía PRISMA-P, se realizó la búsqueda en tres bases de datos, Scielo, EBSCO y LILACS, con las palabras clave de "salud", "agrícola", "agricultura", en idioma español; "health", "agriculture", "agricultural" en idioma inglés y "saúde", "agrícola" para el idioma portugués, con la respectiva definición de criterios de inclusión y exclusión. Resultados: Se obtuvo 65 artículos en los que se identificó como riesgo ocupacional (R0) prevalente, el riesgo químico seguido del riesgo ergonómico y biológico (contacto con animales), Brasil y Colombia tienen mayor número de publicaciones en torno al tema. Conclusiones: Se realiza una aproximación a los contextos rurales en relación a riesgos laborales y a su vez con la importancia de comprensión desde una mirada estructural de los determinantes de la salud, los cuales se convierten en desafíos para promover la atención de la salud pública.

Palabras clave: Agricultura; riesgos laborales; salud laboral; condiciones de trabajo. (Fuente: DeCS, Bireme).

\begin{abstract}
Introduction: International Labour Organization (ILO) guidelines promote inter-sectorial alliances in areas such as health and safety in agricultural sector jobs. Objective: To identify labor risks and their health consequences in Latin American workers by means of a systematic review covering the 2010 - 2020 period. Materials and methods: A search in the Scielo, EBSCO, and LILACS databases was conducted using the PRISMA-P guide. The keywords used were: "salud" "agrícola", and "agricultura" in Spanish; "health" "agriculture", and "agricultural" in English; and "saúde" "agrícola" in Portuguese. Proper definitions of inclusion and exclusion criteria were included. Results: A total of 65 articles were retrieved, identifying the prevalence of several occupational risks (OR) such as chemical risks and ergonomic and biological risks (animal contact), which were classified as the first and second most prevalent risks, respectively. Brazil and Colombia have the largest number of publications in reference to this topic. Conclusions: An understanding of the rural context in relation to the labor risks was accomplished. Likewise, the importance of the comprehension of health determinants from a structural point of view is highlighted, which have become challenges in promoting public health care.
\end{abstract}

Keywords: Farming; occupational risks; occupational health; working conditions. (Source: DeCS, Bireme).

\footnotetext{
*Autor de correspondencia

Johana Madelyn Matabanchoy Salazar

e-mail: udenarmade7@udenar.edu.co
} 


\section{Introducción}

La Organización Internacional del Trabajo (OIT)(1), menciona que el sector agrícola corresponde aproximadamente a un tercio de la fuerza de trabajo mundial, ya sea como trabajadores agrícolas asalariados en empresas e industrias, agricultores comerciales (grandes, pequeños), trabajadores ocasionales o jornaleros, trabajadores a tiempo parcial o trabajadores sub-contratados ${ }^{(2,3)}$. Lo anterior, evidencia la existencia de heterogeneidad en las modalidades de trabajo en la economía rural, que da cuenta de la importancia de que las políticas hagan la distinción acerca de las personas que cuentan con ocupación regular -por ende de medidas de protección-y de quienes a menudo están excluidos de la cobertura de la legislación laboral nacional (trabajadores ocasionales o jornaleros, subcontratados o a tiempo parcial)(4).

Para el sector agrícola en el año 2014, la OIT(1) estimó que las cifras de accidente de trabajo y enfermedad laboral, representaban 6.300 fallecimientos al día y más de 2.3 millones al año; para el año 2019 añadió que aproximadamente la mitad de los 321.000 accidentes mortales anuales en el mundo se presentan en la agricultura(4).

El Convenio 184 sobre la seguridad y salud en la agricultura(5), refiere que la prevención de los accidentes y enfermedades laborales debe partir de un proceso de identificación, evaluación y seguimiento de los factores de riesgo relacionados con el trabajo y el hecho de que los trabajadores conozcan los riesgos existentes, las condiciones relacionadas en el lugar de trabajo que pueden afectar a la salud o la seguridad, se constituye en un derecho(3).

Cabe mencionar que, el término riesgo, se entiende como aquellos peligros probables, latentes que giran en torno al daño(6). Adicional a lo anterior, Bauman(7), menciona tres tipos de peligros: a) como amenaza al organismo; b) en relación a la seguridad de los medios de vida (empleo -renta, supervivencia para el caso de invalidez o vejez) y; c) con el lugar que ubica a la persona en el mundo (posición jerárquica e identidad).

Entre los factores de riesgo mayoritariamente incidentes en la agricultura, se encuentran los relacionados con el uso de maquinaria, levantamiento de carga pesada (trastornos del sistema osteomuscular), exposición a condiciones meteorológicas y atmosféricas (temperatura, polvo, lluvia, radiación solar) y factores de riesgo químico por el manejo de plaguicidas, entre otros ${ }^{(5)}$. Sin embargo, emerge la importancia de generar procesos de identificación de nuevos riesgos en el lugar de trabajo y abordar enfermedades ocupacionales relacionadas como por ejemplo, con el aspecto psicosocial $(5,8)$, las cuales son menos reconocidas en el contexto(9).

Desde la Agenda 2030 para el Desarrollo Sostenible(10), se menciona que el trabajo decente se articula con las condiciones de trabajo seguras, incluyendo aspectos desde la Seguridad y Salud en el Trabajo (SST), permitiendo el reconocimiento no sólo de la calidad del trabajo sino también de la dignidad humana en marco de la cultura preventiva de la seguridad y salud(8,11,12). Es de resaltar que el promover la protección de SST es una tarea primordial en la adopción de entornos de trabajo seguros y saludables, puesto que, en las zonas rurales los niveles de pobreza son generalmente más altos que en las urbanas, los ingresos suelen ser bajos y las condiciones de trabajo deficientes, entre ellas, el no disponer de instalaciones básicas que provean de agua, saneamiento y atención de salud(3).

Sin embargo, la seguridad y salud en el trabajo en el sector agrícola a pesar de ser ampliamente divulgada como aspecto prioritario de atención en el marco de la salud pública internacional no se han identificado revisiones sistemáticas que den cuenta de un panorama actual e integral sobre los riesgos presentes en las condiciones de trabajo para el sector y sus consecuencias asociadas. Es así como se presentan revisiones de tema, sistemáticas y críticas(13-16) prioritariamente enfocadas en la comprensión del riesgo químico (manejo y consecuencias) dejando de lado una mirada integral frente a los diferentes riesgos, por ello, el objetivo de la presente revisión sistemática fue identificar los riesgos ocupacionales (RO) y las consecuencias en la salud de trabajadores latinoamericanos del sector agrícola desde una revisión sistemática en el periodo 2010- 2020, con el propósito de ser de aporte para futuros procesos de investigación, así como también la generación de reflexiones contextualizadas desde las dinámicas de trabajo latinoamericanas, en marco de seguridad y salud en el trabajo agrícola. 


\section{Materiales y métodos}

La unidad de análisis se integró por 65 estudios (Figura 1) siguiendo la matriz de registro documental basada en el protocolo PRISMA-P(17) que parte de: a) la pregunta de investigación; b) la delimitación del objetivo; c) el establecimiento de criterios de inclusión y exclusión; d) el plan de búsqueda; e) los artículos que cumplieron los criterios de inclusión fueron codificados según nomenclatura de consecutivo (año, base de datos y país de publicación); f) la identificación de artículos duplicados; g) la revisión a profundidad y exclusión de artículos; h) el análisis y sistematización de las categorías previamente delimitadas correspondientes al año de publicación, título, idioma, tipo de ingreso, nivel de escolaridad, tipo de cultivo, tipo de riesgo, consecuencias para la salud y tipo de estudio y; por último i) la interpretación y análisis de resultados.

La revisión sistemática integró las bases de datos Scielo, EBSCOhost y LILACS, seleccionadas por su reconocimiento frente a estudios empíricos en salud. Es de mencionar que para cada base de datos se tuvo en cuenta los parámetros de búsqueda integral. Como criterios de inclusión se establecieron: a) ventana de publicación entre los años 2010 - 2020; b) artículos que incluyeran en el título, el resumen o en las palabras clave los términos: "salud", "agrícola", "agricultura" para el idioma español; "health", "agriculture", "agricultural" para el idioma de inglés y "saúde", "agrícola" para el idioma de portugués; c) investigaciones realizadas en países latinoamericanos. Es de mencionar que las palabras clave establecidas se escogieron para abarcar una mayor cobertura de artículos empíricos en las bases de datos mencionadas, no se elige como criterio de búsqueda el término "riesgo" puesto que en las búsquedas preliminares, el uso de este, arrojaba pocos estudios asociados a la temática, por ello, se decidió ampliar al uso de categorías como "salud" y "agricultura" y en la etapa de lectura a profundidad, identificar los riesgos descritos por los investigadores.

Como criterios de exclusión: a) estudios que no sean empíricos; b) que no cuenten con metodología o resultados; c) la temática de investigación no se relacione con RO o consecuencias para la salud y; d) población que no corresponda a trabajadores del sector agrícola.

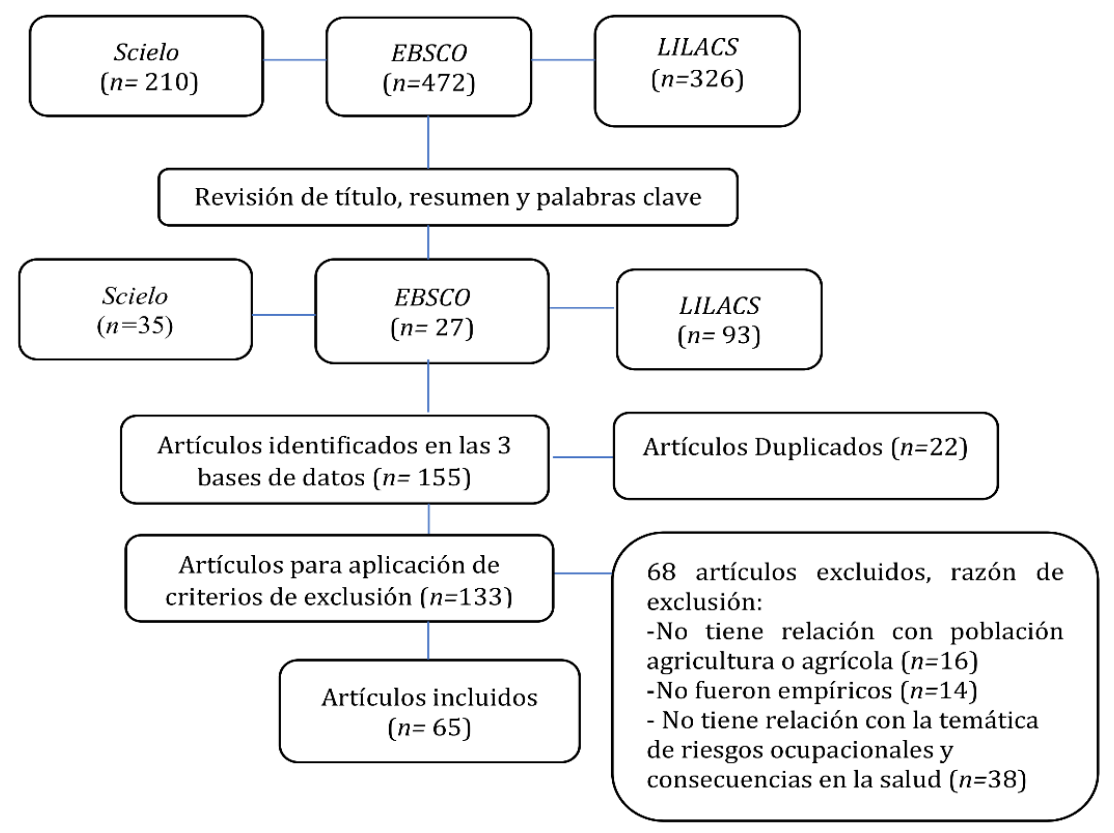

Figura 1. Diagrama de flujo del proceso de selección de estudios según guía PRISMA(17)

La matriz integró los siguientes aspectos: código, título original, autores, año, idioma, referencia, país, tipo de cultivo, tipo de estudio, técnicas e instrumentos de recolección de información, población de estudio, tipos de riesgos (químico, psicosocial, biológico, condiciones meteorológicas, mecánico, ergonómico, otros), consecuencias para la salud. La búsqueda y recopilación de información se 
realizó integrándola a través del gestor bibliográfico Mendeley, su búsqueda correspondió al periodo de octubre a diciembre del año 2020(18). Posteriormente, un experto en revisiones sistemáticas corroboró la precisión de las etapas desarrolladas, de esta manera, se consolidó el análisis y discusión de la información(19), se retomó aspectos de unidades de registro desde temáticas o categorías previamente delimitadas y reglas de numeración que integró aspectos de frecuencia y presencia.

Con respecto a la evaluación de riesgo de sesgo, se buscó minimizar errores en la selección de estudios a fin de cumplir el objetivo propuesto, y se excluyó aquellos que no se adecuaban a los criterios de revisión establecidos con el propósito de identificar coherencia en método, objetivo y diferentes apartados de los artículos revisados, de esta manera se otorgó validez y confianza. El equipo investigador realizo la revisión individual y confirió concepto favorable o desfavorable.

\section{Consideraciones éticas}

La presente investigación se enmarca desde aspectos éticos previstos la Resolución 8430 de 1993(20) para investigaciones en salud en seres humanos, con el propósito de conocer relaciones entre causas y enfermedad y aportar así a la prevención y control. Así mismo, velando los principios éticos (beneficencia, justicia) con el propósito de presentar información coherente con el objeto de estudio(21).

\section{Resultados}

\section{Año y país de publicación}

A partir de la revisión a profundidad, el 63\% de los estudios se realizaron en el periodo de 2016 a 2020, evidenciándose un incremento en publicaciones; correspondientes al año $2017(20 \% n=13)^{(22,23,32,33,24-}$ 31); $2016(15 \% n=10)^{(34-43)} ; 2019(11 \% n=7)^{(44-50)}$ $2018(8 \% n=5)^{(51-55)}$ y; $2020(8 \% n=5)^{(56-60)}$. De los 65 estudios, los años con menor publicación se ubicaron entre el 2011 y 2012 con 2 artículos respectivamente (3\%) (Tabla 1$)$.

Con respecto a los países con mayor representatividad de publicación se encontró Brasil con un $38 \%(n=25)$, seguido de Colombia con $25 \%$ $(n=16)$ y México 9\% $(n=6)$. Los países con menor incidencia en publicación fueron Chile, Costa Rica, El Salvador con un artículo y el 62\% $(n=40)$ correspondió al idioma español, seguido de portugués $22 \%(n=14)$ e inglés $17 \%(n=11)$ (Tabla 1$)$.

\section{Población}

\section{Tipo de ingreso}

La mayoría de estudios no refieren el tipo de ingreso, sin embargo, aquellos que si lo describen, en su mayoría prevalece como tipo de ingreso el jornal(27,28,48,52,61-63), seguido de pequeña producción o micro productor $(35,36,47,48,56,64)$; agricultura empresarial $(34,64,65)$; productores en $\operatorname{arriendo}^{(47,63)}$ y; agricultura familiar(23,35,45).

\section{Nivel de escolaridad}

Es importante mencionar que no todos los estudios explicitan la información sobre el nivel de formación de los participantes. De los estudios que explicitan este aspecto, se evidenció que la mayoría de participantes cuentan con nivel de escolaridad mayormente de primaria incompleta, seguido de formación a nivel de secundaria incompleta $(36,47,59,63,64,66,67)$ y estudios que reportan nivel superior de formación educativa, es bajo.

Tabla 1. Publicaciones relacionadas con RO y consecuencias para salud según país y año

\begin{tabular}{|c|c|c|c|c|c|c|c|c|c|c|c|c|}
\hline País/Año & 2011 & 2012 & 2013 & 2014 & 2015 & 2016 & 2017 & 2018 & 2019 & 2020 & $\mathbf{n}$ & $\%$ \\
\hline Brasil & 1 & 1 & 1 & 8 & 1 & 4 & 5 & 1 & 2 & 1 & 25 & 38 \\
\hline Colombia & 1 & 1 & 2 & 2 & 2 & 1 & 3 & 2 & & 2 & 16 & 25 \\
\hline México & & & 1 & & & 2 & 1 & & 2 & & 6 & 9 \\
\hline Argentina & & & & & & & 2 & 1 & 1 & 1 & 5 & 8 \\
\hline Uruguay & & & & & & 1 & & 1 & & & 2 & 3 \\
\hline Bolivia & & & & 1 & & & 1 & & & & 2 & 3 \\
\hline Chile & & & & & 1 & & & & & & 1 & 2 \\
\hline Costa Rica & & & & & 1 & & & & & & 1 & 2 \\
\hline Ecuador & & & & & & & 1 & & & 1 & 2 & 3 \\
\hline El Salvador & & & & & & & & & & 1 & 1 & 2 \\
\hline Paraguay & & & & & & 1 & & & 1 & & 2 & 3 \\
\hline Venezuela & & & & & 1 & 1 & & & & & 2 & 3 \\
\hline Total & 2 & 2 & 4 & 11 & 6 & 10 & 13 & 5 & 6 & 6 & 65 & 100 \\
\hline
\end{tabular}




\section{Tipo de cultivos}

Los tipos de cultivos que más sobresalen en los estudios encontrados son: el de caña de azúcar(35,41,47,48,68-72); seguido de maíz $(28,37,45,46,63,68,73,74) ; \quad \quad \operatorname{soja}^{(37,45,46,56,75)} \quad$ y; tabaco $(40,45,59,76)$; en menor proporción: café, yuca, arroz, banano, flores, pitahaya, coliflor, repollo, durazno, arveja, haba, cebolla, lechuga, papa, cebada, algodón, tomate, yerba mate, entre otros.

\section{Tipo de estudio}

Artículos que mencionan el tipo de estudio correspondieron a 55, de los cuales el tipo de estudio cualitativo fue predominante con un $60 \%(n=33)$, seguido del tipo de estudio cuantitativo $38 \%(n=21)$ y mixto $2 \%(n=1)$. Entre las técnicas o instrumentos de recolección de información, se encontró: entrevistas estructuradas y semiestructuradas (26\%); entrevistas a profundidad (5\%); cuestionarios (15\%); observación directa y no participante (12\%); encuestas (15\%) y; grupo focal (8\%). Entre las de menos frecuencia se observó, toma de muestras biológicas, estudios de caso, registro fotográfico y taller participativo.

\section{Riesgos ocupacionales y consecuencias para la salud}

En relación a los tipos de riesgos en la agricultura, prevaleció el riesgo químico con un $74 \% \quad(n=48)$; ergonómico 32\% ( $n=21)$; biológico $22 \% \quad(n=14)$; condiciones climáticas y atmosféricas $22 \%(n=14)$; condiciones y organización de trabajo $18 \%(n=12)$ y accidentes por uso de herramientas manuales y uso de máquinas 9\% (n=6) (Tabla 2).

Para el riesgo biológico, se determinó contacto con animales como serpientes, escorpiones, arañas, garrapatas, alacrán y plagas $(35,38,47,53,61,70,77)$, se ha evidenciado que la percepción de este riesgo se enfoca hacia una baja severidad y vulnerabilidad, puesto que autores como Peña, et al.(47), mencionan que los trabajadores no distinguen como riesgo la picadura de garrapatas, de igual forma lo reportaron Villegas, et al.(78) quienes refieren que la picadura del alacrán se concibe como un suceso cotidiano. Adicional a lo anterior, en cuanto a las medidas de prevención como, el uso de guantes, es bajo y se evita transitar por senderos o zonas que haya la infestación del vector(53,78).

En relación a la manipulación de sustancias químicas, cabe resaltar que en estudios se ha identificado una baja percepción de riesgo, o la negación del mismo como un factor que incida en la salud $(60,66)$, lo que ha conllevado al no uso de los Elementos de Protección Personal (EPP) $(23,49,51,60,66)$. Por otra parte, estudios reportan que los EPP con mayor frecuencia de uso corresponden son camisetas de manga larga, gorra o sombrero, pantalón largo, botas altas. Sin embargo, no se cuenta con traje especial, es bajo el uso de guantes, mascarilla o careta, permitiendo un contacto directo con la piel y vías respiratorias $(46,63,79)$. Con respecto al manejo de sustancias durante la jornada de trabajo, se evidencia que no se realiza un cambio de ropa habitual al finalizar la jornada de fumigación y se consume alimentos durante la aplicación(25).

En relación a la capacitación para el manejo, almacenamiento y uso de químicos, algunos estudios refieren que este aspecto no se contempla o no existe claridad(49,60,63,79), sumado a los bajos niveles de educación que dificultan la lectura de las fichas técnicas de dichos productos $(60,63)$; de manera que la elección de plaguicidas (dosis, periodicidad y forma de aplicación) se lleva a cabo a partir de la experiencia intergeneracional -tradición oral-(48,80). No obstante, Tabares y López(63), determinaron que las empresas proveedoras suministran información de nuevos productos con el propósito de comercializarlos, y autores como Polanco, et al.(46) resaltan la importancia promover programas para la agricultura sustentable.

Por último, como elementos emergentes, se evidenciaron determinantes sociales de la salud, como la educación, nivel de formación donde prevalece una baja alfabetización funcional, puesto que la mayoría la población reporta primaria incompleta o hasta nivel de secundaria, influyendo en una falta de comprensión de fichas técnicas, por otra parte, se reporta una alta intoxicación por manejo de sustancias químicas, sin embargo, la condición económica para acceder a un EPP no está al alcance de los ingresos que se obtienen del trabajo agrícola, en función de formas de ocupación precarias desde condiciones artesanales de producción, producción agraria mínimamente tecnificada, no afiliación a seguridad social, jornadas extensas, escaso tiempo para el descanso e hidratación mínima, falta de estabilidad laboral, y las condiciones de temperatura ambiente para el desarrollo de trabajo agrícola(47,57,59,63,81). 
Tabla 2. Riesgos ocupacionales y consecuencias para la salud

\begin{tabular}{|c|c|}
\hline Riesgo ocupacional & Consecuencia para la salud \\
\hline $\begin{array}{l}\text { Riesgo químico por manipulación de sustancias } \\
\text { químicas }(22,23,44-46,48,51,52,54,56,58,62,25,63,67,73,79,82-86,26-30,37,41) \\
\text { Plaguicidas }\end{array}$ & $\begin{array}{l}\text { Intoxicación (cefalea, náuseas, dolor de estómago, vómito, dolor abdominal, dificultad } \\
\text { para respirar) } \\
\text { Malformaciones congénitas } \\
\text { Alergias } \\
\text { Alteraciones neurológicas } \\
\text { Enfermedades dermatológicas } \\
\text { Cáncer } \\
\text { Enfermedades de vías respiratorias } \\
\text { Enfermedades gastrointestinales } \\
\text { Enfermedades auditivas } \\
\text { Enfermedades oftalmológicas } \\
\text { Pérdidas fetales } \\
\text { Trastornos inmunológicos } \\
\text { Problemas reproductivos } \\
\text { Alteración en inmunidad }\end{array}$ \\
\hline $\begin{array}{l}\text { Riesgo ergonómico }(23,51,87,52,54,55,58,59,62,65,79) \\
\text { Levantamiento de carga pesada } \\
\text { Movimientos repetitivos e intensos (rotación) } \\
\text { Movimientos por minutos en tareas específicas (cosecha de } \\
\text { rosas) } \\
\text { Posturas estáticas de trabajo o forzadas } \\
\text { Horarios de colocación y descanso } \\
\text { Sobreesfuerzo físico }\end{array}$ & $\begin{array}{l}\text { Enfermedades músculo esqueléticas } \\
\text { Fatiga visual } \\
\text { Agotamiento físico } \\
\text { Dolor lumbar } \\
\text { Dolor torácico } \\
\text { Dolor cervical } \\
\text { Tendinitis } \\
\text { Dolor postural } \\
\text { Dolor muscular } \\
\text { Fatiga crónica } \\
\text { Síndrome del túnel del carpo } \\
\text { Deterioro de columna vertebral }\end{array}$ \\
\hline $\begin{array}{l}\text { Riesgo biológico }(38,39,42,47,53,59,86) \\
\text { Contacto con animales (serpientes, escorpiones, arañas, } \\
\text { garrapatas, alacrán, plagas) } \\
\text { Contacto con ríos, lagunas profundas que puedan presentarse } \\
\text { contaminantes microbiológicos (bacterias o micotoxinas, } \\
\text { metales pesados no autorizados, hongos) } \\
\text { Contacto agua estancada, material contaminado como clavos y } \\
\text { tornillos oxidados } \\
\text { Contacto con vegetación, como espinas } \\
\text { Ambientes contaminados de gases de circulación de camiones, } \\
\text { fábricas o de residuos. }\end{array}$ & $\begin{array}{l}\text { Infección respiratoria } \\
\text { Enfermedades Zoonóticas } \\
\text { Picaduras y mordeduras } \\
\text { Enfermedad Rickettsiosis } \\
\text { Enfermedad tabaco verde } \\
\text { Esporotricosis Humana (Sporothrix Schenckii) } \\
\text { Blastomicosis Sudamericana, Paracoccidioidomicosis (PMC) o enfermedad de Lutz- } \\
\text { Splendore }\end{array}$ \\
\hline $\begin{array}{l}\text { Condiciones climáticas y atmosféricas }(47,51,52,58,86) \\
\text { Diversidad climática (radiación solar, humedad, frío excesivo, } \\
\text { lluvia) y temperaturas extremas }\end{array}$ & $\begin{array}{l}\text { Deshidratación } \\
\text { Calambres } \\
\text { Pérdida de electrolitos } \\
\text { Desmayos } \\
\text { Náuseas } \\
\text { Neumonía } \\
\text { Impacto nocivo de temperaturas extremas }\end{array}$ \\
\hline $\begin{array}{l}\text { Riesgo Mecánico }(52,54,79) \\
\text { Manipulación de maquinaria (segadora) } \\
\text { Manipulación herramientas (Machete, poda de cizalla, hoces, } \\
\text { rastrillo) } \\
\text { Caída a nivel por uso de palas } \\
\text { Caída de objetos }\end{array}$ & $\begin{array}{l}\text { Accidentes con herramientas manuales } \\
\text { Accidentes por uso de maquinaría } \\
\text { Fracturas por uso de machetes, poda de cizallas y hoces } \\
\text { Esguinces }\end{array}$ \\
\hline $\begin{array}{l}\text { Condiciones y organización del Trabajo } \mathbf{o}^{(22,23,54,55,59,65,74)} \\
\text { Jornadas de trabajo extensas (12 horas) } \\
\text { Alta demanda de atención } \\
\text { Ritmo acelerado de producción } \\
\text { Sobrecarga de trabajo } \\
\text { Trabajo monótono y repetitivo } \\
\text { Relación con el jefe } \\
\text { Inestabilidad contractual } \\
\text { Violencia de género } \\
\text { Acoso laboral } \\
\text { Discriminación } \\
\text { Falta de claridad de roles } \\
\text { Relaciones en el trabajo } \\
\text { Flexibilidad y baja remuneración económica (pago a destajo, } \\
\text { subcontratación, contrato por tiempo de cosecha) } \\
\text { Ausencia de normas contractuales } \\
\text { Baja autonomía }\end{array}$ & $\begin{array}{l}\text { Frustración del trabajo en relación a la satisfacción y motivación del trabajo agrícola } \\
\text { Estrés en temporadas de cosecha, o ante alta demanda } \\
\text { Grado severo de estrés } \\
\text { Conducta suicida (ideación, intento y suicido) } \\
\text { Trastornos mentales comunes (depresión, ansiedad) } \\
\text { Sentimientos de inutilidad } \\
\text { Baja autoestima } \\
\text { Cambios de humor (enojo, irritabilidad, nerviosismo) } \\
\text { Desesperanza por el futuro }\end{array}$ \\
\hline
\end{tabular}




\section{Discusión}

En la revisión sistemática se evidenció la predominancia del riesgo químico y sus afecciones en la salud desde una perspectiva más biologicista y unicausal de la enfermedad, así como también, los factores ergonómicos y de las condiciones de trabajo, que tienen implicaciones en la calidad de vida de las personas. Sin embargo, no emergieron con la misma intensidad, los aspectos psicosociales en el trabajo y en la vida de las personas ${ }^{(88,89)}$.

Consecuentemente al amplio número de estudios en relación a la manipulación y exposición a plaguicidas en el sector agrícola, se evidenció múltiples afectaciones a la salud de los trabajadores como: dolor de cabeza, náuseas, vómito, erupciones, desorientación, efectos dermatológicos, gastrointestinales, carcinógenos, respiratorios, neurológicos, entre otros; similar a lo reportado en otros estudios(14,90,91), situación que devela la necesidad de control sanitario y la revisión de tecnologías empleadas para la producción en la agricultura ${ }^{(92)}$ para disminuir la exposición al riesgo en salud, en contraposición de las prácticas convencionales(64,93).

Adicional a lo anterior, se evidencia que se reporta una baja percepción con respecto a la severidad y susceptibilidad frente al riesgo, al respecto autores como Kim, et al.(94), mencionan que los trabajadores perciben estos síntomas como aspectos que hacen parte del trabajo agrícola y no como un riesgo ocupacional, por lo tanto, se naturalizan los síntomas asociados a la intoxicación. Esta misma situación coincide con Palacio y Paz, quienes registraron en el 2011 que el $70 \%$ de aplicadores de plaguicidas no contaban con EPP, el $54 \%$ refirió ingerir sus alimentos en el mismo lugar de la aplicación -cultivosy la gran mayoría afirmó bañarse entre 30 minutos y dos horas después de la jornada de trabajo, mientras que una quinta parte lo hacía en el canal del agua junto a los sembrados $(90,95)$, reflejo de una subestimación del riesgo que puede provocar su apatía u optimismo irracional(96).

Dicha sobrestimación del riesgo, no solo se debe al bajo nivel de escolaridad de los trabajadores agrícolas lo cual se reporta en diferentes estudios $(36,44,47,59,63,66,67)$, sino también se evidenció que se relaciona con aspectos culturales y procesos de capacitación frente al manejo y exposición de los mismos; con respecto al primero, autores como
Damalas y Koutroubas(97), y Jin, et al.(98) coinciden en que la toma de decisiones frente al uso de plaguicidas que enfrentan los trabajadores agrícolas así como el uso de EPP, están determinadas no sólo por el conocimiento de las consecuencias hacia la salud y sino también de los aspectos culturales, económicos y políticos estableciéndose en el sector agrícola marcos de acción aprendidos y moldeados en el tiempo, los cuales definen umbrales aceptables de riesgo.

Con respecto al segundo, se evidenció baja asesoría, asistencia técnica, capacitación y formación por parte de casas proveedoras de insumos y autoridades municipales tanto sanitarias como ambientales, por lo tanto, resulta una prioridad el establecimiento de estrategias de prevención primaria y secundaria en la zona rural(63,99), que involucre diferentes actores intersectoriales $(15,91,100,101)$. Adicional, Mendes y Roncalli(24), expresan que el bajo de los fumigadores, puede presentarse por el interés de lucro del sistema productivo y comercial, quedando en segundo plano las estrategias de educación y prevención, hecho que a su vez incrementa la vulnerabilidad de la exposición.

Díaz y Rentería(6) mencionan que las concepciones de salud en el trabajo han transformado su enfoque, pasando de los accidentes de trabajo, las lesiones y afectaciones físicas, a la integración de elementos relacionados con la forma de trabajo y la salud mental. Al respecto, Sotomayor ${ }^{(9)}$ manifiesta que las investigaciones en temas psicosociales son menos reconocidas en el contexto de agricultores, no obstante, refiere la existencia de casos de diagnóstico de depresión y reporte de suicidio en el sector agrícola, que pueden desencadenarse por la interrupción en la producción laboral y consecuente pérdida del sustento.

Así mismo, Faria, et al.(102), manifiestan que las causas externas de mayor mortalidad para los agricultores están relacionadas con accidentes laborales y el suicidio, este último, fue prevalente en relación a la ingesta de plaguicidas(103), sin embargo, existe la necesidad de profundizar en estudios que determinen la multicausalidad de conducta suicida en este sector(104-106).

Perió y Prieto(107) expresan que los contextos de trabajo están integrados por las condiciones ambientales, temporales, de demanda, carga de trabajo y condiciones sociales, que deben caracterizarse en el marco de empleo estable, 
desempleo, empleo precario, trabajo informal, entre otros, para determinar así, la exposiciones a riesgo (posibilidad de daño) en el lugar de trabajo y el modo como están organizadas, tanto en la gestión como en el control del trabajo, las tareas y la tecnología(108). Asimismo, plantea la importancia de reconocer la influencia de las condiciones de trabajo materiales y psicosociales, de manera individual o conjunta.

Con respecto al trabajo rural(90), es relevante tener en cuenta que aspectos de tiempo y espacialidad varían dependiendo del sector productivo (caña, arroz, tabaco, papa, floricultura, entre otros), los cuales demandan diferentes ritmos según la etapa de producción en que se encuentre(109), así como también, prevalece el trabajo manual(110) y en algunas regiones existen diferencias de actividades según el sexo, por ejemplo, Ferreira et al.(31), reportan una sobrecarga de trabajo en mujeres al integrar labores domésticas y del campo (cría animales, cultivo de hortalizas, artesanías); organización de trabajo en elementos como contratos a destajo, exposiciones a altas temperaturas, radiación solar, extensas jornadas laborales(111); baja afiliación al sistema de riesgos laborales, seguridad social(63,111,112), índices de pobreza, que conjuntamente con los bajos ingresos(113) obstaculizan la compra de EPP(78).

Estudios han realizado avances con respecto a indagar y promover alternativas para la agricultura sostenible y así reducir la exposición a plaguicidas desde diferentes opciones ecológicas, que permita no solo la sostenibilidad ambiental sino también el bienestar social, considerando la salud humana como un aspecto esencial y de prioridad, requiriendo así el apoyo gubernamental para la transición(91,101,114-116).

Algunas poblaciones latinoamericanas se encuentran en un estado de vulnerabilidad hacia su salud en relación a determinantes sociales dado el bajo nivel de educación, bajo ingreso económico, bajo acceso a servicios de salud y protección social, bajo desarrollo, implementación y seguimiento de políticas que promuevan aspectos de protección integral a la seguridad y salud en el sector agrícola, así como aspectos de trabajo informal y precario. Por ejemplo, el acceso a salud es una dimensión desigual entre las zonas rurales y urbanas(113,117,118), así como el saneamiento básico y electricidad en algunas áreas(119); siendo necesario, realizar un seguimiento a las condiciones de trabajo y la calidad de vida, en específico profundizar en las condiciones psicosociales(108).
Las condiciones psicosociales, son aquellas que repercuten en la vida a través de procesos de estrés que "superan los límites soportables [...] cuando se producen cambios fisiopatológicos y de conducta"(108), lo cual se relaciona con las condiciones de empleo y trabajo; ellas no son directamente mesurables. Estas condiciones abarcan exigencias relacionadas con la organización (ritmo de trabajo, complejidad del trabajo, carga mental y emocional, autonomía, relaciones interpersonales, apoyo social, recompensas, incertidumbre, toma de decisiones, justicia, seguridad laboral, atención, entre otras)(120) y para finalizar Ruiz et al.(121), mencionan que las trayectorias ocupacionales de las personas se entrecruzan con las trayectorias vitales, así la calidad de vida y trabajo compromete no sólo el presente y sino también el futuro de las sociedades.

\section{Recomendaciones}

Es importante incluir una mirada desde los determinantes sociales en función del contexto y país; así como estudios empíricos que den cuenta de las condiciones de trabajo en contexto de emergencia sanitaria por COVID-19, desde una perspectiva multifactorial y dinámica del proceso saludenfermedad. Por último, es necesario profundizar es la comprensión psicosocial en el contexto laboral y la incidencia en la salud ocupacional en el sector agrícola.

\section{Conclusiones}

Se realiza una aproximación a los contextos rurales en relación a riesgos laborales y a su vez con la importancia de comprensión desde una mirada estructural de los determinantes de la salud, los cuales se convierten en desafíos para promover la atención de la salud pública.

Conflicto de intereses: Ninguno declarado por los autores.

\section{Referencias}

1. Organización Internacional del Trabajo. Tendencias Mundiales del Empleo 2014: ¿Hacia una recuperación sin creación de empleos? [Internet]. 2014 [consultado 2021 Mar 25]. Disponible en: https://www.ilo.org/global/research/globalreports/global-employment-trends/2014/lang-es/index.htm

2. Organización Internacional del Trabajo. Trabajo decente y productivo en la agricultura. 2015. Disponible en: https://www.ilo.org/wcmsp5/groups/public/--ed_dialogue/--sector/documents/publication/wcms_437214.pdf

3. Organización Internacional del Trabajo. Transición a la formalidad en la economía rural informal [Internet]. 2019. Disponible 
https://www.ilo.org/wcmsp5/groups/public/---

ed_dialogue/---

sector/documents/publication/wcms_437218.pdf

4. Organización Internacional del Trabajo. Los derechos en el trabajo en la economía rural, trabajo decente en la economía rural notas de orientación de políticas [Internet]. 2019. Disponible en: http://www.ilo.org/global/topics/economicand-social-development/rural-

development/WCMS\%7B\%5C_\%7D437227/lang-es/index.htm

5. Oficina Internacional del Trabajo. El Convenio No. 184 sobre la seguridad y Salud en la Agricultura y su ratificación. Guía 3 [Internet]. 2001. Disponible en: https://www.ilo.org/dyn/normlex/es/f?p=NORMLEXPUB: 12100:0::NO::P12100_ILO_CODE:C184

6. Díaz F, Rentería E. De la seguridad al riesgo psicosocial en el trabajo en la legislación colombiana de salud ocupacional. Estud Socio-Jurídicos [Internet]. 2017;19(2):129-55. DOI: 10.12804/revistas.urosario.edu.co/sociojuridicos/a.4981

7. Bauman Z. Miedo líquido La sociedad contemporánea y sus temores [Internet]. Cambridge, RU: Polity Press; 2006. Disponible

en: https://redpaemigra.weebly.com/uploads/4/9/3/9/49391 489/bauman_zygmunt__-miedo_liquido.pdf

8. Organización Mundial de la Salud. Convenio sobre el marco promocional para la seguridad y salud en el trabajo [Internet]. Núm. 187. 2006. Disponible en: https://www.ilo.org/dyn/normlex/es/f?p=NORMLEXPUB: 12100:0::NO::P12100_INSTRUMENT_ID:312332

9. Sotomayor JU. $16^{\circ}$ Reunión Interamericana a Nivel Ministerial en Salud y Agricultura (RIMSA 16): AgriculturaSalud-Medio Ambiente: sumando esfuerzos para el bienestar de los pueblos de las Américas. Santiago, Chile: Organización Panamericana de la Salud; 2012. Disponible en: http://www.panaftosa.org/rimsa16/dmdocuments/RIMSA 16(INF4)\%20esp.pdf

10. Naciones Unidas. Informe de los objetivos de Desarrollo Sostenible [Internet]. 2019. Disponible en: https://unstats.un.org/sdgs/report/2019/The-SustainableDevelopment-Goals-Report-2019_Spanish.pdf

11. Oficina Internacional del Trabajo. Trabajar juntos para promover un medio ambiente de trabajo seguro y saludable. 2017.

12. Ribeiro M. Contribuições da psicologia para repensar o conceito de trabalho decente. Rev Psicol Organ Trab [Internet]. 2020 [consultado 2021 Mar 25];20(3):1114-21. Disponible http://pepsic.bvsalud.org/pdf/rpot/v20n3/v20n3a09.pdf

13. Barbosa $\mathrm{P}$, Aguilar $\mathrm{H}$. Trabalho rural e riscos à saúde: uma revisão sobre o "uso seguro" de agrotóxicos no Brasil. Ciência \& Saúde Coletiva [Internet]. 2014 Oct;19(10):4197-208. Disponible

https://www.scielo.br/j/csc/a/ynd3LjKy44N9KJSLrqSZQR $\mathrm{m} /$ abstract/?lang=pt

14. Campuzano Cortina C, Feijoó Fonnegra LM, Manzur Pineda K, Palacio Muñoz M, Rendón Fonnegra J, Zapata Díaz JP. Efectos de la intoxicación por glifosato en la población agrícola: revisión de tema. CES Salud Pública [Internet]. 2017 Nov 8 [consultado 2021 Oct 9];8(1):121-33. Disponible en: https://revistas.ces.edu.co/index.php/ces_salud_publica/ar ticle/view/4427

15. Trujillo-Abella I, Orjuela-Yacué C. Principales causas de muerte de agricultores en Colombia periodo 2010-2016. Rev la Univ Ind Santander Salud [Internet]. 2020 Feb;52(1):41-
9.

Disponible

en:

https://revistas.uis.edu.co/index.php/revistasaluduis/articl e/view/10302/10208

16. Rodrigues Selmi G da R, Zanaga Trapé A. Proteção da saúde de trabalhadores rurais: a necessidade de padronização das metodologias de quantificação da exposição dérmica a agrotóxicos. Cad Saude Publica [Internet]. 2014 [consultado 2021 Oct 9];30(5):952-60. Disponible en: http://www.scielo.br/j/csp/a/4mhQ3LZHR4d7QwCc9LhG Bgy/abstract/?lang=pt

17. Moher D, Shamseer L, Clarke M, Ghersi D, Liberati A, Petticrew M, et al. Preferred reporting items for systematic review and meta-analysis protocols (PRISMA-P) 2015 statement. Rev Esp Nutr Humana y Diet [Internet]. 2016;20(2):148-60. Disponible en: https://systematicreviewsjournal.biomedcentral.com/articl es/10.1186/2046-4053-4-1

18. Gough D, Sandy O, Thomas J. An Introduction to Systematic Reviews [Internet]. 2nd ed. SAGE; 2012 [consultado 2021 Oct 12]. Disponible en: https://books.google.com.co/books?id=41sCDgAAQBAJ\&pr intsec $=$ frontcover\&dq=an+introduction+to+systematic + revi ews+david+gough+pdf\&hl=es-

419\&sa $=X \&$ redir_esc $=y \# v=$ onepage $\& \mathrm{q}=$ an introduction to systematic reviews david gough pdf\&f=false

19. Bardin L. Análisis de contenido. 3ra ed. Madrid: Ediciones Akal; 2002. Disponible en: https://books.google.com.co/books?id=IvhoTqll_EQC\&print $\mathrm{sec}=$ frontcover $\& \mathrm{dq}=$ analisis $+\mathrm{de}+$ contenido+Bardin.pdf\&hl= es\&sa=X\&redir_esc=y\#v=onepage \&q\&f=false

20. Ministerio de Salud. Resolución 8430 de 1993. 1993 Oct 4. Disponible

en: https://www.minsalud.gov.co/sites/rid/Lists/BibliotecaDi gital/RIDE/DE/DIJ/RESOLUCION-8430-DE-1993.PDF

21. Congreso de la República. Ley 1090 [Internet]. Sep 6, 2006. Disponible en: http://www.psicologiaprospectiva.com/introley1090.html

22. García C, Breilh J, de Lorudes Larrea M. La interacción entre la exposición a agrotóxicos y componentes relevantes del sistema inmune en comunidades de La Paz Bolivia: una mirada desde la epidemiología crítica. Rev Fac Cienc Méd Univ Cuenca. 2017;35(2):39-47. Disponible en: https://publicaciones.ucuenca.edu.ec/ojs/index.php/medic ina/article/view/1721

23. Vitali S. Precariedad en las condiciones de trabajo y salud de los trabajadores del sector bananero del Ecuador. Salud trab (Maracay). 2017;25(1):9-22. Disponible en: https://dialnet.unirioja.es/servlet/articulo? codigo $=638480$ 7

24. Mendes Araújo IM, Roncalli da Costa Oliveira ÂG. Agronegocio y agrotóxicos: impactos sobre la salud de los trabajadores agrícolas en el Noreste Brasileño. Trab Educ e Saúde [Internet]. 2017;15(1):117-29. Disponible en: https://www.scielo.br/j/tes/a/Ny5PpLyDMmSJbhNc8CBfK Vf/abstract/?lang=es

25. Rojas Rodríguez AE, Toro-Osorio BM, Díaz-Zapata JA. Niveles de colinesterasa sérica en caficultores del Departamento de Caldas, Colombia. Rev Salud Pública. 2017;19(3):318-24. Disponible http://www.scielo.org.co/pdf/rsap/v19n3/0124-0064rsap-19-03-00318.pdf

26. Gutiérrez Lesmes OA, Loboa Rodriges NJ, Plata Casas LI. Situación epidemiológica de la intoxicación por sustancias químicas en el departamento del Meta-Colombia, periodo 
2009-2014. Rev Biosalud [Internet]. 2017 Jun;16(1):30-42. DOI: 10.17151/biosa.2017.16.1.5.

27. Ortega Martínez LD, Martínez Valenzuela C, Waliszewski S, Ocampo Mendoza J, Huichapan Martínez J, El Kassis E, et al. Nivel tecnológico de invernadero y riesgo para la salud de los jornaleros. Rev Electrónica Nov Sci. 2017;9(18):21-42. DOI: $10.21640 /$ ns.v9i18.730.

28. Amador C, Luna Rondón JM, Puello Alcocer EC. Prácticas empleadas por fumigadores de plaguicidas del medio y bajo Sinú departamento de Córdoba. Temas Agrar. 2017;22(1):29-40. Disponible en: https://revistas.unicordoba.edu.co/index.php/temasagrari os/article/view/913

29. Machado AL, Butinof M, Eandi MA, Portilla AM, Fernandez RA, Soria V, et al. Vulnerabilidad y riesgo por plaguicidas en horticultura del cinturón verde en Córdoba, Argentina. Rev Fac Nac Salud Pública. 2017;35(1):99-110. Disponible en: http://www.scielo.org.co/pdf/rfnsp/v35n1/0120-386Xrfnsp-35-01-00099.pdf

30. Díaz SM, Sánchez F, Varona M, Eljach V, Muñoz MN. Niveles de colinesterasa en cultivadores de papa expuestos ocupacionalmente a plaguicidas, Totoró, Cauca. Rev la Univ Ind Santander Salud [Internet]. 2017 Mar;49(1):85-92. DOI: 10.18273/revsal.v49n1-2017008.

31. Ferreira Leite J, Dimenstein M, Bezerra Dantas C, Lucas Silva E, Sales Macedo JP, Pereira de Sousa A. Condições de vida, saúde mental e gênero em contextos rurais: um estudo a partir de assentamentos de reforma agrária do Nordeste brasileiro: uma revisão sistemática. Av en Psicol Latinoam [Internet]. 2017 May;35(2):301-16. DOI: 10.12804/revistas.urosario.edu.co/apl/a.4768.

32. Componogara S. Implicações do uso de agrotóxicos: percepções de familiares de crianças portadoras de neoplasia. Rev Pesqui Cuid é Fundam [Internet]. 2017 [consultado 2021 Mar 31];9(3):786-94. Disponible en: https://www.redalyc.org/pdf/5057/Resumenes/Resumen_ 505754116023_1.pdf

33. Casaril dos Santos Cargnin M, Echer IC, Rosa da Silva D. Fumicultura: uso de equipamento de proteção individual e intoxicação por agrotóxico Tobacco farming: use of personal protective equipment and pesticide poisoning. Rev Pesqui Cuid é Fundam Online [Internet]. 2017 Apr 11 [consultado 2021 Mar 31];9(2):466-72. Disponible en: http://www.seer.unirio.br/index.php/cuidadofundamental /article/view/5444

34. Haeffner R, Heck RM, da Rosa Jardim VM. Prevalência de agravos de pele e fatores associados em trabalhadores de uma empresa agropecuária do sul do Brasil. Rev Bras Med do Trab [Internet]. 2016;14(3):214-21. Disponible en: https://www.rbmt.org.br/details/114/pt-BR/prevalenciade-agravos-de-pele-e-fatores-associados-em-trabalhadoresde-uma-empresa-agropecuaria-do-sul-do-brasil

35. Príncipe Nunes DM, Saturnino da Silva M, Meira Cordeiro R. Work and risk experience among Northeastern migrant works in sugarcane plantations in the state of São Paulo, Brazil. Saúde e Soc [Internet]. 2016;25(4):1122-35. Disponible en: https://www.scielo.br/j/sausoc/a/n65VjZ797BCSRvFcdSF WBXS/?format=pdf\&lang=en

36. Cezar-Vaz ME, Bonow C, Almeida de Mello M, Santos da Silva MR. Socio-environmental approach in nursing: focusing on rural labor and the use of pesticides. Rev Bras Enferm. 2016;69(6):1114-21. DOI: 10.1590/0034-7167-2016-0364.

37. Heinzen J, Rodríguez N. Procesos destructores para la salud vinculados a la manipulación de agroquímicos en trabajadores agrícolas de Young, Uruguay. Cienc \& Trab [Internet]. 2016;18(56):117-23. DOI: 10.4067/S071824492016000200007.

38. Cuellar Gordo LC, Amador Orozco B, Olivares Goenaga G, Borré Ortiz YM, Pinedo Otálvaro J. Comportamiento epidemiológico del accidente ofídico en el departamento del Magdalena, Colombia (2009-2013). Ciencias la Salud [Internet]. 2016 Jul;14(2):161-77. DOI: 10.12804/revsalud14.02.2016.02.

39. Araujo P, Aguilar G, Arce N, Martínez M. Paracoccidioidomicosis detectados en el período 2004-2013 en el Laboratorio Central de Salud Pública de Asunción Paraguay. Rev del Nac. 2016;8(2):62-71. Disponible en: https://pesquisa.bvsalud.org/portal/resource/pt/biblio884758

40. Riquinho DL, Hennington ÉA. Sistema integrado de produção do tabaco: saúde, trabalho e condições de vida de trabalhadores rurais no Sul do Brasil. Cad Saude Publica [Internet]. 2016;32(12):1-10. Disponible en: https://www.scielo.br/j/csp/a/RhFwsYSn5cKVgKLCkDZKY jq/abstract/?lang=pt

41. Guzmán-Plazola P, Guevara-Gutiérrez RD, Olguín-López JL, Mancilla-Villa OR. Perspectiva campesina, intoxicaciones por plaguicidas y uso de agroquímicos. Idesia (Arica) [Internet]. 2016 Jun;34(3):69-80. DOI: 10.4067/S071834292016000300009.

42. González SK, García LA, Flores DE, Perez RE, Luis-León JJ, Mota-Alvarez J, et al. Infección por Sporothrix schenckii en agricultores del caserío "Peñón de Gabante": Municipio Tovar. Estado Aragua. Venezuela. Comunidad y Salud. 2016;14(2):24-32. Disponible en: http://ve.scielo.org/scielo.php?script=sci_arttext\&pid=S16 90-32932016000200004

43. Aranda $P$, Castro Vásquez $M$ del C. El campo de la agroindustria en el noroeste de México y la salud de sus jornaleras: una propuesta de estudio. Salud Colect [Internet]. 2016 Mar;12(1):55-70. DOI: 10.18294/sc.2016.878.

44. Flores L, Gamarra G, Gonzáles de Bóveda H, Paredes M. Monitoreo comunitario para la vigilancia de exposición al uso de plaguicidas en Paraguay, Año 2018. Rev Salud Pública Parag. 2019;9(1):9-18. DOI: 10.18004/rspp.2019.junio.918.

45. Landini F, Beramendi M, Vargas GL. Uso y manejo de agroquímicos en agricultores familiares y trabajadores rurales de cinco provincias argentinas. Rev argent salud publica. 2019;10(38):22-8. Disponible en: https://pesquisa.bvsalud.org/portal/resource/pt/biblio996334

46. Polanco Rodríguez AG, Magaña Castro TV, Cetz Iuit J, Quintal López R. Uso de agroquímicos cancerígenos en la región agrícola de Yucatán, México. Cent Agrícola. 2019;46(2):7283. Disponible en: http://scielo.sld.cu/scielo.php?script=sci_arttext\&pid=S025 3-57852019000200072

47. Peña Ríosa Y, López OE, Borrero Ramírez Y. Caracterización de los determinantes sociales de las Rickettsiosis en El Jigual, Rosas, Cauca en el año 2016. Un estudio de caso cualitativo. Gerenc y Políticas Salud [Internet]. 2019 Nov;18(37):1-32. DOI: 10.11144/Javeriana.rgps18-37.cdsr.

48. Ramírez-Mora E, Pérez-Vásquez A, Landeros-Sánchez C, Martínez-Dávila JP, Villanueva-Jiménez JA, Lagunés Espinoza LC. Occupational exposure to pesticides in sugarcane agroecosystems in the central region of Veracruz state, 
Mexico. Rev Bio Ciencias. 2019;6:e495. DOI: 10.15741/revbio.06.e495.

49. Miranda C, de Oliveira RM. Utilização de agrotóxicos no assentamento três pontes, Município de Perolândia (go): fatores de risco á saúde. Rev Geográfica América Cent [Internet]. 2019 Jun;2(63):369-85. DOI: 10.15359/rgac.632.14 .

50. Schumacker Brust R, de Oliveira L, Silva A, Regazzi I, Aguiar G, Knupp V. Epidemiological profile of farmworkers from the state of Rio de Janeiro. Rev Bras Enferm [Internet]. 2019 Feb;72(Suppl 1):122-8. Disponible en: https://www.scielo.br/j/reben/a/xtRLTs6qXhYkYz7DZgV MntS/abstract/?lang=es

51. Gordon Morante C, Marrugo Negrete JL. Prácticas agrícolas y riesgos a la salud por el uso de plaguicidas en agrícultores subregión Mojana - Colombia. Rev Investig Agrar y Ambient [Internet]. $2018 \quad$ Feb;9(1):29-40. DOI: 10.22490/21456453.2098.

52. Linardelli MF. Entre la finca, la fábrica y la casa: el trabajo productivo y reproductivo de trabajadoras agrícolas migrantes en Mendoza (Argentina) y su incidencia en la salud-enfermedad. Salud Colect [Internet]. 2018;14(4):75777. DOI: 10.18294/sc.2018.1395.

53. Manosalva-Sánchez C, Zuleta-Dueñas LP, Castañeda-Porras O. Estudio descriptivo del accidente ofídico, CasanareColombia, 2012-2014. MedUNAB [Internet]. 2018 Jul;20(3):338-48. DOI: 10.29375/01237047.2672.

54. Nión Celio S, Pereyra V. Construcción social del riesgo en el agro uruguayo: desafíos a la actividad sindical. Salud Colect [Internet]. 2018;14(4):743-55. Disponible en: http://revistas.unla.edu.ar/saludcolectiva/article/view/13 85

55. Cezar-Vaz MR, Alvez Bonow C, Gautério Abreu D, Vaz J, Almeida de Mello M, Modernel Xavier D. Carga de trabajo rural y factores asociados al uso de medicamentos por personas ancianas. Rev Esc Enferm USP. 2018;52:e03374. DOI: $10.1590 /$ S1980-220X2017048303374.

56. Kunin J, Lucero PA. Percepción social del riesgo y dinâmicas de género en la producción agrícola basada en plaguicidas en la pampa húmeda Argentina. Sex Salud y Soc (Rio Janeiro) [Internet]. 2020;(35):58-81. DOI: 10.1590/19846487.sess.2020.35.04.a.

57. Orantes Navarro CM, Almaguear López M, Galbán PA, Díaz Amaya M, Hernández S, Herrera Valdés R, et al. La epidemia de enfermedad renal crónica en El Salvador: la influencia de los agroquímicos. Rev Cubana Med Trop. 2020;72(2):e531. Disponible en: http://scielo.sld.cu/pdf/mtr/v72n2/15613054-mtr-72-02-e531.pdf

58. Litardo-Velásquez CA, Real-Pérez GL, Cedeño-Macías LA, Rodríguez-Coveña KL, Hidalgo-Ávila AA, Zambrano-Mero RA. Prevención de Riesgos Laborales en el cultivo de Pitahaya, Manabí, Ecuador. Ing Ind. 2020;41(2):e4113. Disponible en: http://scielo.sld.cu/pdf/rii/v41n2/18155936-rii-41-02-e4113.pdf

59. Gastal Fassa A, Spada Fiori N, Meucci Carvalho R, Müller Xavier N, Peres de Carvalho M. Dolor cervical entre agricultores que producen tabaco en el sur de Brasil. Salud Colect [Internet]. 2020 Jul;16:e2307. DOI: 10.18294/sc.2020.2307.

60. Lopez de Mesa Y. The decision-making process of synthetic pesticide use in agricultural communities in Colombia: a grounded theory approach. Rev Fac Nac Salud Pública [Internet]. $2020 \quad$ Mar;38(2):1-7. DOI: 10.17533/udea.rfnsp.e331277.
61. de Oliveira JA, Moraes Nina S de F. Environment and health of working women: transformations in a community in the Brazilian Amazon. Saúde e Soc. 2014;23(4):1162-72. DOI: 10.1590/S0104-12902014000400004.

62. Camarena Ojinaga L, von Glascoe C, Martínez Váldes C, Arellano García E. Riesgos del trabajo y salud: percepción de mujeres indígenas jornaleras en el noroeste de México. Salud Colect. 2012;9(2):247-56. Disponible en: https://www.scielosp.org/pdf/scol/2013.v9n2/247256/es

63. Tabares JC, López YL. Salud y riesgos ocupacionales por el manejo de plaguicidas en campesinos agricultores, municipio de Marinilla, Antioquia, 2009. Rev Fac Nac Salud Pública [Internet]. 2011;29(4):432-44. Disponible en: https://www.redalyc.org/pdf/120/12021522018.pdf

64. Gamarra MG, Tullo E, Salinas Z, Flores L, Paredes M, González $\mathrm{H}$, et al. Implementación de herramienta tecnológica (TIC) para la vigilancia de factores ambientales y posibles afecciones relacionadas a la exposición por uso de plaguicidas agrícolas en Paraguay. Rev salud publica del Paraguay [Internet]. 2019 Jun;9(1):19-32. DOI: 10.18004/rspp.2019.junio.19-32.

65. Valero Pinto AM, Vergara Quezada J, Rojas Cortés CM, Quiceno Hurtado LM. Condicionantes de género, empleo y trabajo y su posible vínculo con afecciones y dolencias musculoesqueléticas y psicosociales de temporeras frutícolas de packing agroindustrial de la Región del Libertador General Bernardo O’Higgins, Chile. Cienc \& Trab. 2015;17(53):107-14. Disponible en: https://scielo.conicyt.cl/pdf/cyt/v17n53/art03.pdf

66. Tofolo C, Meneghello Fuentefria A, Moreira Farias F, Mansur Machado M, Souza Oliveira LF. Contributing factors for farm workers' exposure to pesticides in the west of the state of Santa Catarina, Brazil. Acta Sci Heal Sci [Internet]. 2014 Oct;36(2):153-9. 10.4025/actascihealthsci.v36i2.22383.

67. Varona Uribe M, Castro R, Paéz MI, Carvajal N, Barbosa E, León LM, et al. Impacto en la salud y el medio ambiente por exposición a plaguicidas e implementación de buenas prácticas agrícolas en el cultivo de tomate, Colombia, 2011. Rev Chil Salud Pública [Internet]. 2012;16(2):96-106. Disponible en: https://revistasaludpublica.uchile.cl/index.php/RCSP/artic le/view/20267/21435

68. Pignati W, Pereira Oliveira N, Cândido da Silva AM. Vigilância aos agrotóxicos: quantificação do uso e previsão de impactos na saúde-trabalho-ambiente para os municípios brasileiros. Ciência \& Saúde Coletiva [Internet]. 2014;19(12):4669-78. DOI: $10.1590 / 1413-812320141912.12762014$.

69. Galiano A, Vettorassi A, Navarro VL. Trabalho, saúde e migração nos canaviais da região de Ribeirão Preto (SP), Brasil: o que percebem e sentem os jovens trabalhadores? Rev Bras Saúde Ocup [Internet]. 2012 Jun;37(125):51-64. Disponible en: https://www.scielo.br/j/rbso/a/CkGpMQ8X6DLfdntXrKzQ 4Qm/abstract/?lang=pt

70. Borges dos Santos JC, Azevedo Hennington É. Aqui ninguém domina ninguém: sentidos do trabalho e produção de saúde para trabalhadores de assentamento do Movimento dos Trabalhadores Rurais Sem Terra. Cad Saude Publica [Internet]. 2013;29(8):1595-604. DOI: 10.1590/0102311 X00096612.

71. Ferreria Ceccato AD, de Carvalho Junior LC, Campos Cuissi R, Monteschi M, Galvão Oliveira N, Padovani $\mathrm{CR}$, et al. 
Absenteísmo por doença ocupacional de trabalhadores rurais no setor canavieiro. Cad Saude Publica [Internet]. 2014 Oct;30(10):2169-76. DOI: 10.1590/0102$311 X 00026413$.

72. Aredes Priuli RM, de Moraes MS, Morais Chiaravalloti R. The impact of stress on the health of sugar cane cutters. Rev Saúde Pública. 2014;48(2):225-31. Disponible en: https://pubmed.ncbi.nlm.nih.gov/24897043/

73. Bustamante Villaroel S, Segales Rojas DJ, Zurita Herrera L, Fernandez Arancibia M, Torrico Condarco S, Jarro Mena R. Uso inadecuado de plaguicidas y sus consecuencias en la salud de la población La Villa, Punata, Cochabamba, Bolivia, 2013. Gac Médica Boliv. 2014;37(1):11-4. Disponible en: http://www.scielo.org.bo/pdf/gmb/v37n1/v37n1_a03.pdf

74. Puello Alcocer EC, Ortega Montes JE, Valencia Jiménez NN. Factores sociales asociados a la salud de los trabajadores informales agrícolas de la ciudad de Montería - Córdoba, Colombia. Rev Univ salud. 2013;15(2):103-12. Disponible en: http://www.scielo.org.co/pdf/reus/v15n2/v15n2a02.pdf

75. Nasrala Neto E, de Castro Lacaz FA, Pignati WA. Health surveillance and agribusiness: the impact of pesticides on health and the environment. Danger ahead! Ciência \& Saúde Coletiva. 2014;19(12):4709-18. DOI: 10.1590/1413812320141912.03172013.

76. Vaz Guimarães P, de Carvalho F, Câmara MC, Fernandes de Brito P, Barrios S, Bottom M, et al. Lesões Cutâneas PréMalignas em Residentes de um Município Rural do Rio Grande do Sul, Brasil. Rev Bras Cancerol. 2014;60(3):22330. Disponible

en: https://rbc.inca.gov.br/revista/index.php/revista/article/v iew/467

77. Cândia Veiga JP, Machado Trevisani D, Makishi F, Caixeta de Abreu M, Pacheco e Silva M, Alvesi Zacareli M. Padrões de saúde e segurança no trabalho e extrativismo: o caso de comunidades rurais da Amazônia brasileira. Saúde e Soc [Internet]. 2017 Sep;26(3):774-85. DOI: 10.1590/S010412902017166075.

78. Villegas-Arrizón A, Garzón-Mayo R, Flores-Moreno M, Andersson N. El uso de guantes como factor protector contra picaduras de alacrán durante la pizca de maíz en el estado de Guerrero, México. Salud Publica Mex. 2009;51(2):126-33. Disponible

en: http://www.scielo.org.mx/pdf/spm/v51n2/v51n2a08.pdf

79. Agudelo $R$, Soto $M$, Pérez $M$, Jaramillo $M$, Moreno N. Condiciones de vida y trabajo de familias campesinas agricultoras de Marinilla, un pueblo agrario del oriente Antioqueño, Colombia. Rev Fac Nac Salud Pública. 2011;31(3):319-28.

80. Góngora-Gómez AM, García-Ulloa M, Muñoz-Sevilla NP, Domínguez-Orozco AL, Villanueva-Fonseca BP, HernándezSepúlveda JA, et al. Heavy-metal contents in oysters (Crassostrea gigas) cultivated on the southeastern coast of the Gulf of California, Mexico. Hidrobiológica [Internet]. 2017;27(2):219-27.

DOI: 10.24275/uam/izt/dcbs/hidro/2017v27n2/Garcia.

81. Kjellstrom T, Crowe J. Climate Change, Workplace Heat Exposure, and Occupational Health and Productivity in Central America. Int J Occup Environ Health. 2011 Jul;17(3):270-81. Disponible en: https://pubmed.ncbi.nlm.nih.gov/21905396/

82. Miranda-Contreras L, Cruz I, Osuna JA, Gómez-Pérez R, Berrueta L, Salmen S, et al. Efectos de la exposición ocupacional a plaguicidas sobre la calidad del semen en trabajadores de una comunidad agrícola del estado Mérida, Venezuela. Invest Clin. 2015;56(2):67-78. Disponible en: http://ve.scielo.org/scielo.php?script=sci_arttext\&pid=S05 35-51332015000200003

83. Vargas Trejos Y. Exposición a agroquímicos y creencias asociadas a su uso en la cuenca hidrográfica del Río Morote, Guanacaste, Costa Rica: un estudio de casos. Cienc \& Trab. 2015;17(52):54-68. Disponible en: https://scielo.conicyt.cl/pdf/cyt/v17n52/art11.pdf

84. Portilla-Portilla Á, Pinilla-Monsalve GD, Caballero-Carvajal AJ, Gómez-Rodríguez E, Marín-Hernández LR, ManriqueHernández EF, et al. Prevalencia de signos y síntomas asociados a la exposición directa a plaguicidas neurotóxicos en una población rural colombiana en 2013. Medicas UIS. 2014;27(2):41-9. Disponible en: http://www.scielo.org.co/pdf/muis/v27n2/v27n2a05.pdf

85. Morera LJ, Villamil DA, Zambrano M. Relación de los niveles de formación y conductas adoptadas en la manipulación de plaguicidas por unas poblaciones agricultoras de Colombia. CES Salud Públic. 2015;6(2):116-21. Disponible en: https://revistas.ces.edu.co/index.php/ces_salud_publica/ar ticle/view/3353

86. Goyeneche-Ortegón RL, Jiménez-Sánchez YAC. Dos miradas sobre el riesgo laboral: cultivadores de papa del Municipio de Toca, Boyacá, Colombia. Ciencias la Salud. 2015;13(2):25970. DOI: 10.12804/revsalud13.02.2015.09.

87. Barrero LH. Ergonomía en floricultura en Colombia: resultados y lecciones. Rev Cienc Salud. 2014;12:45-53. DOI: 10.12804/revsalud12.esp.2014.06.

88. Renteria Pérez E. Psicología(s) organizacional(es) y del (de los) trabajo(s). Programa Editorial Universidad del Valle; $2020 . \quad$ Disponible https://www.jstor.org/stable/j.ctv15pjzc4

89. Garrido-Pinzón J, Uribe-Rogríguez AF. RIESGOS PSICOSOCIALES DESDE LA PERSPECTIVA DE LA CALIDAD DE VIDA LABORAL. Acta Colomb Psicol [Internet]. 2011 [consultado 2021 Mar 25];14(2):27-34. Disponible en: http://www.scielo.org.co/pdf/acp/v14n2/v14n2a03.pdf

90. Chávez J, Bravo Velásquez E, Fierro Bósques M. Percepción de la salud por parte de los agricultores en zonas de producción agrícolas dedicadas al monocultivo de maíz duro. Caso Cantón Ventanas, Provincia de Los Ríos-Ecuador. YACHANA Rev Científica [Internet]. 2017 [consultado 2021 Mar 31];6(1):25-36. Disponible en: http://revistas.ulvr.edu.ec/index.php/yachana/article/vie $\mathrm{w} / 361 / \mathrm{pdf}$

91. Moreno Dos Reis M, Natividade de Oliveira AP, Baretto Turci S, Maciel Dantas R, Pinto da Sila V, Gross C, et al. Knowledge, attitudes, and practices of women farmers concerning tobacco agriculture in a municipality in Southern Brazil. Cad Saude Publica [Internet]. 2017 [consultado $2021 \mathrm{Apr}$ 1];33(Suppl 3):e00080516. DOI: 10.1590/0102311X00080516.

92. Moreira de Oliveira K. Controle sanitário de agrotóxicos no Brasil: o caso do Metamidofós. Rev Cad Ibero-Am Direito Sanitário. 2016;5(2):159-75. DOI: doi.org/10.17566/ciads.v5i2.291.

93. Ammar AS. Nanotechnologies associated to floral resources in agri-food sector. Acta Agronómica [Internet]. 2018;67(1):148-61. DOI: 10.15446/acag.v67n1.62011.

94. Kim K-H, Kabir E, Jahan SA. Exposure to pesticides and the associated human health effects. Sci Total Environ [Internet]. 2017;575:525-35. Disponible en: https://linkinghub.elsevier.com/retrieve/pii/S0048969716 
31926X

95. Palacios-Nava ME, Paz-Román M. Sintomatología persistente en trabajadores agrícolas expuestos a plaguicidas organofosforados. Rev Fac Nac Salud Pública [Internet]. 2011;29(2):153-62. Disponible en: https://www.scielosp.org/article/spm/1999.v41n1/55$61 /$

96. Carbonel-Siam AT, Torres-Valle A. Evaluación de percepción de riesgo ocupacional. Ing Mecánica [Internet]. 2010;13(3):18-25. Disponible http://scielo.sld.cu/pdf/im/v13n3/im03310.pdf

97. Damalas C, Koutroubas S. Farmers' Training on Pesticide Use Is Associated with Elevated Safety Behavior. Toxics [Internet]. 2017;5(3):19. DOI: 10.3390/toxics5030019.

98. Jin J, Wang W, He R, Gong H. Pesticide Use and Risk Perceptions among Small-Scale Farmers in Anqiu County, China. Int J Environ Res Public Health [Internet]. 2017;14(1):29. DOI: 10.3390/ijerph14010029.

99. Gómez JP. Accidente por animales ponzoñosos y venenosos: su impacto en la salud ocupacional en Colombia. Rev Fac Nac Salud Pública [Internet]. 2011;29(4):419-31. Disponible en: https://www.redalyc.org/articulo.oa?id=12021522008

100. Zambrano Ospina AM. Accidente ofídico como evento de interés en salud publica en Colombia: Aporte al diseño de estrategias de gestión [Trabajo de grado]. Bogotá, D.C.: Universidad Nacional de Colombia; 2012. Disponible en: https://www.researchgate.net/publication/280804172_Ac cidente_ofidico_como_evento_de_interes_en_salud_publica_e n_Colombia_Aporte_al_diseno_de_estrategias_de_gestion

101. Haby M, Soares A, Chapman E, Clark R, Korc M, Galvão L. Interventions that facilitate sustainable development by preventing toxic exposure to chemicals: an overview of systematic reviews. Rev Panam Salud Publica. 2016;39(6):378-86. Disponible en: https://pubmed.ncbi.nlm.nih.gov/27706431/

102. Faria N, Fassa A, Meucci R. Association between pesticide exposure and suicide rates in Brazil. Neurotoxicology. 2014 Dec;45:355-62. Disponible en: https://www.sciencedirect.com/science/article/pii/S0161 813X14000849

103. Rabasquinho C, Pereira H. Género e saúde mental: Uma abordagem epidemiológica. Análise Psicológica [Internet]. 2007;25(3):439-54. DOI: 10.14417/ap.456.

104. Rodrigues G. Intoxicações e óbitos por agrotóxicos no Estado de Goiás, Brasil e inovações legislativas. Cad Ibero Am Direito Sanit [Internet]. 2018;7(1):229-49. DOI: 10.17566/ciads.v7i1.471.

105. Brito Teixeira JR, de Oliveira Ferraz CE, Ferreira Couto JC, Alves Nery A, Casotti CA. Intoxicações por agrotóxicos de uso agrícola em estados do Nordeste brasileiro, 1999-2009. Epidemiol e Serviços Saúde [Internet]. 2014 Sep;23(3):497$508 . \quad$ Disponible en: https://www.scielo.br/j/ress/a/rWGrMgJyXPZcsq85NQfTC $6 \mathrm{~m} /$ abstract/?lang=pt

106. Zambrano Ochoa V, Manrique Rubiano KM, Medina Sánchez K, Medina Sánchez K. DEPRESIÓN Y EXPOSICIÓN OCUPACIONAL A PLAGUICIDAS: UNA REVISIÓN DE ALCANCE DE LA LITERATURA 2010 a 2020 [Trabajo de grado]. Bogotá D.C: Universidad del Rosario; 2020 [consultado 2021 Apr 1]. Disponible en: https://repository.urosario.edu.co/bitstream/handle/1033 6/30726/ARTICULO\%20Valery\%20Zambrano\%20Karen\% 20Manrique $\% 20$ Kimberlyn $\% 20$ Medina $\% 202020 \% 2012 \% 2$ 009.pdf? sequence $=4 \&$ isAllowed $=y$
107. Peiró JM, Prieto F. Tratado de psicología del trabajo. Vol. II: Aspectos psicosociales del trabajo [Internet]. Editorial Síntesis S.A; $2002 . \quad$ Disponible en: https://www.sintesis.com/psicologia-social-171/tratadode-psicologia-del-trabajo-vol-ii-aspectos-psicosociales-deltrabajo-libro-1448.html

108. Benach JM, Muntaner C. Empleo, Trabajo Y Desigualdades en Salud: Una visión Global [Internet]. Icaria editorial; 2010 [consultado 2021 Mar 25]. Disponible en: https://www.researchgate.net/publication/318361793_Em pleo_Trabajo_Y_Desigualdades_en_Salud_Una_vision_Global

109. Pereira Rocha L, Cezar-Vaz MR, de Almeida M, Borges A, Santos da Silva M, Sena-Castanheira J. Workloads and occupational accidents in a rural environment. Texto \& Context - Enferm [Internet]. 2015 Jun;24(2):325-35. DOI: 10.1590/0104-07072015000480014.

110. Rojas M, Gimeno D, Vargas-prada S, Benavides FG. Dolor musculoesquelético en trabajadores de América Central: resultados de la I Encuesta Centroamericana de Condiciones de Trabajo y Salud. Rev Panam Salud Publica. 2015;38(2):120-8. Disponible en: https://iris.paho.org/handle/10665.2/10046

111. Varona ME, Díaz SM, Briceño L, Sánchez-Infante CI, Torres $\mathrm{CH}$, Palma RM, et al. Determinantes sociales de la intoxicación por plaguicidas entre cultivadores de arroz en Colombia. Rev Salud Publica [Internet]. 2016 Jul;18(4):61729. DOI: 10.15446/rsap.v18n4.52617.

112. Picciotto S, Hertz-Picciotto I. Commentary: healthy worker survivor bias: a still-evolving concept. Epidemiology [Internet]. $2015 \quad$ Mar;26(2):213-5. DOI: 10.1097/EDE.0000000000000233.

113. Osorio-Quintero L, Lopera-García LD, López-Arango YL, Rendón-Ospina ID, Tabares-López JC, Medina-Tamayo M, et al. Condiciones de trabajo y de seguridad social en asociaciones de pequeños $y$ medianos agricultores campesinos con prácticas de economía solidaria en tres municipios del oriente antioqueño, Colombia, 2015. Rev Fac Nac Salud Pública [Internet]. 2019 May;37(2):36-48. Disponible http://www.scielo.org.co/pdf/rfnsp/v37n2/0120-386Xrfnsp-37-02-36.pdf

114. Castelãoa Tetila E, Cecílio Tetila JL, Pistori H, Ferreira da Silva MA. Desafios do modelo de desenvolvimento agrícola do estado de Mato Grosso do Sul: uma proposta para o desenvolvimento sustentável. Interações (Campo Gd) [Internet]. $2020 \quad$ Sep;21(3):615-32. DOI: 10.20435/inter.v21i3.2430.

115. García-Céspedes D, Lima-Cazorla LA, Ruiz-Gutierrez L, Santana-Romero J, Calderón-Peñalver PA. Agroecosistemas con probables riesgos a la salud por contaminación con metales pesados. Rev Cuba Química. 2016;28(1):378-93. Disponible http://scielo.sld.cu/pdf/ind/v28n1/ind04116.pdf

116. Polanco-López Y, Hernández-Carmona D, Escobar-Péres ML, Aguirre-Acevedo DC, Parra-Hernández Á. Medición de niveles de colinesterasas eritrocitarias en agricultores usuarios de plaguicidas y en practicantes de agroecología, San Cristóbal, Medellín, Colombia. Rev Fac Nac Salud Pública [Internet]. 2019 [consultado 2021 Apr 1];37(3):25-33. Disponible en: https://revistas.udea.edu.co/index.php/fnsp/article/view/ 334048/20794742

117. Martins Arruda N, Gori Maia A, Correia Alves L. Desigualdade no acesso à saúde entre as áreas urbanas e rurais do Brasil: 
uma decomposição de fatores entre 1998 a 2008. Cad Saude Publica [Internet]. 2018 [consultado 2021 Apr 1];34(6):e00213816. DOI: 10.1590/0102-311X00213816.

118. Garnelo L, Lima J, Carvalho Rocha E, Herkrath FJ. Acesso e cobertura da Atenção Primária à Saúde para populações rurais e urbanas na região norte do Brazil. Saúde em Debate [Internet]. 2018 Sep;42:81-99. DOI: 10.1590/010311042018 S106.

119. Morais V, Ferreira J, Miranda Cruz J, Brito da Silva L, Castellá Sarriera J. Pobreza multidimensional e seus aspectos subjetivos em contextos rurais e urbanos nordestinos. Estud
Psicol [Internet]. 2016;21(2):146-56. DOI: 10.5935/16784669.20160015.

120. Cirilo Neto M, Dimenstein M. Saúde Mental em Contextos Rurais: o Trabalho Psicossocial em Análise. Psicol Ciência e Profissão [Internet]. 2017 Jun;37(2):461-74. DOI: 10.1590/1982-3703002542016.

121. Ruiz Frutos C, Delclòs J, García AM, Benavides FG. Salud laboral: Conceptos y técnicas para la prevención de riesgos laborales [Internet]. 3 ed. España: Elsevier España; 2006. Disponible en: 